\title{
Le service médical aux colons : gestation et implantation d'un service infirmier au Québec (1932-1943)
}

\section{Johanne Daigle et Nicole Rousseau}

Volume 52, numéro 1, été 1998

URI : https://id.erudit.org/iderudit/005379ar

DOI : https://doi.org/10.7202/005379ar

Aller au sommaire du numéro

Éditeur(s)

Institut d'histoire de l'Amérique française

ISSN

0035-2357 (imprimé)

1492-1383 (numérique)

Découvrir la revue

Citer cet article

Daigle, J. \& Rousseau, N. (1998). Le service médical aux colons : gestation et implantation d'un service infirmier au Québec (1932-1943). Revue d'histoire de l'Amérique française, 52(1), 47-72. https://doi.org/10.7202/005379ar
Résumé de l'article

La crise économique qui a précédé la Deuxième Guerre mondiale a obligé le gouvernement du Québec à donner des terres aux indigents désireux de s'établir dans des régions éloignées des centres urbains. Ce mouvement a donné naissance à de petites communautés appelées " colonies ", composées essentiellement de populations très pauvres. Pour plusieurs raisons, en particulier en raison de contraintes budgétaires, le gouvernement a choisi d'embaucher des infirmières pour répondre à l'ensemble des besoins de santé de ces populations, plutôt que d'y envoyer des médecins. Ce choix l'a conduit à accepter que des infirmières fassent quotidiennement des interventions considérées comme des actes médicaux. C'est ainsi que fut créé un service spécial appelé «Service médical aux colons », consistant en un réseau de plus de cent postes desservis uniquement par des infirmières travaillant en collaboration avec les médecins établis en région. Cet article décrit la longue gestation et l'implantation de ce service méconnu; il démontre que les infirmières ont été au cœur d'un vaste réseau de soins de santé, en particulier pendant une période de grandes difficultés économiques.
Tous droits réservés (C Institut d'histoire de l'Amérique française, 1998
Ce document est protégé par la loi sur le droit d'auteur. L'utilisation des services d'Érudit (y compris la reproduction) est assujettie à sa politique d'utilisation que vous pouvez consulter en ligne.

https://apropos.erudit.org/fr/usagers/politique-dutilisation/ 


\title{
LE SERVICE MÉDICAL AUX COLONS : GESTATION ET IMPLANTATION D'UN SERVICE INFIRMIER AU QUÉBEC (1932-1943) ${ }^{1}$
}

\author{
JOHANNE DAIGLE \\ Département d'histoire \\ Université Laval \\ NICOLE ROUSSEAU \\ Faculté des sciences infirmières \\ Université Laval
}

\section{RÉSUMÉ}

La crise économique qui a précédé la Deuxième Guerre mondiale a obligé le gouvernement du Québec à donner des terres aux indigents désireux de s'établir dans des régions éloignées des centres urbains. Ce mouvement a donné naissance à de petites communautés appelées «colonies», composées essentiellement de populations très pauvres. Pour plusieurs raisons, en particulier en raison de contraintes budgétaires, le gouvernement a choisi d'embaucher des infirmières pour répondre à l'ensemble des besoins de santé de ces populations, plutôt que d'y envoyer des médecins. Ce choix l'a conduit à accepter que des infirmières fassent quotidiennement des interventions considérées comme des actes médicaux. C'est ainsi que fut créé un service spécial appelé «Service médical aux colons», consistant en un réseau de plus de cent postes desservis uniquement par des infirmières travaillant en collaboration avec les médecins établis en région. Cet article décrit la longue gestation et l'implantation de ce service méconnu; il démontre que les infirmières ont été au cœur d'un vaste réseau de soins de santé, en particulier pendant une période de grandes difficultés économiques.

1. Nous remercions le Conseil de recherches en sciences humaines du Canada qui, par son soutien financier, a rendu possible cette recherche. Nos remerciements vont également aux personnes qui ont contribué de diverses façons à sa réalisation: notre collègue Francine Saillant, collaboratrice des débuts de la recherche et à qui on doit l'inspiration originale de ce projet; Ghislaine Hébert, professionnelle de recherche aux ANQ-AT; Guylaine Girouard, David Pankow (département d'histoire), Dominique Boudreau et Manon Henri (Faculté des sciences infirmières), assistants de recherche de l'Université Laval. Nous remercions aussi les évaluateurs anonymes qui nous ont fourni de précieux commentaires. Soulignons enfin que deux partenaires étaient associés à ce projet: l'Ordre des infirmières et infirmiers du Québec et la Corporation du dispensaire de la garde à La Corne, Abitibi.

[1]

RHAF, vol. 52, n 1 , été 1998 


\begin{abstract}
The depression of the 1930s pushed the Government of Québec to give land to indigent persons desirous of settling in Québec's remote regions. This movement created small communities called settlements composed principally of very poor people. For several reasons not the least of which were budgetary constraints, the Government decided to hire nurses to serve the health care needs of these settlements instead of subsidizing doctors. This choice forced the Government to accept the fact that nurses daily did what were essentially considered as medical acts. Thus was created a special service called «Medical Service for Settlers», a network of more than one hundred posts staffed solely by nurses working in collaboration with doctors in the regions. This article describes the long gestation and establishment of this largely unrecognized service; it demonstrates that nurses were at the heart of a vast health care network during the Depression.
\end{abstract}

\title{
INTRODUCTION
}

Dans la foulée des mesures de «secours aux pauvres» mises en œuvre pendant la grande crise économique des années 1930, l'aide à la colonisation agricole a mobilisé d'importants efforts des pouvoirs publics, au Québec plus qu'ailleurs au Canada. En envoyant, souvent en pleine forêt, des milliers de démunis, le gouvernement du Québec devait leur assurer un minimum de services jugés essentiels, en particulier des services de santé. L'interprétation généralement admise d'une mesure transitoire, marginale, voire d'un échec à propos de cette dernière phase intensive de colonisation $^{2}$ ne permet pas de rendre compte d'initiatives novatrices nées des besoins des populations des nouvelles «colonies». L'existence de postes de soins, communément appelés dispensaires ${ }^{3}$ et confiés à des infirmières, a laissé dans la mémoire populaire des souvenirs assez vivaces pour qu'un de ces dispensaires devienne un site historique ${ }^{4}$.

2. Maurice Asselin et Benoît Beaudry-Gourd, dir., L'Abbittibbi et le Témiscaming. Hier et aujourd'hui (Rouyn, Département d'histoire et de géographie, cahier $\mathrm{n}^{\circ} 2$ 2,1975). Voir aussi Michel Pelletier et Yves Vaillancourt, Les politiques sociales et les travailleurs, II: Les années 1930 (Montréal, Publié à compte d'auteur, 1975), 247-266 et Esdras Minville, La législation ouvrière et le régime social dans la province de Québec, Commission royale des relations entre le Dominion et les provinces (Ottawa, Imprimeur du Roi, appendice 5, 1939), 98 p. Paul-André Linteau et al., résument bien ces perspectives dans Histoire du Québec contemporain, 2: Le Québec depuis 1930 (Montréal, Boréal, coll. «Boréal compact», 1989), chapitre 2.

3. Dans le contexte de cet article, le mot fait référence aussi bien au lieu de vie qu'au lieu de travail de l'infirmière; pour cette raison, nous lui préférons l'expression plus précise de «dispensairerésidence» même si, dans une étude sur la sécurité sociale soumise à la Commission royale d'enquête sur les problèmes constitutionnels en 1955, F.-A. Angers affirmait: «Chacun de ces postes est un dispensaire.» F.-A. Angers, La sécurité sociale et les problèmes constitutionnels, Commission royale d'enquête sur les problèmes constitutionnels (Commission Tremblay), 1955, II,3, 112.

4. Il s'agit du dispensaire de La Corne en Abitibi occupé par l'infirmière Gertrude Duchemin de 1936 à 1976; ce site historique a été ouvert officiellement le 24 juin 1997. 
L'ampleur du phénomène et sa persistance bien au-delà de la crise économique, alors que le mouvement de colonisation s'était tari, restent singulièrement méconnues dans l'historiographie ${ }^{5}$. La question de l'accessibilité aux services de santé dans les régions éloignées des centres urbains, envisagée sous l'angle de l'inégale répartition des médecins sur le territoire, ne révèle encore que la pointe de l'iceberg. Bien que les milieux médicaux nord-américains aient pris conscience, dès les années 1920, d'une crise de la médecine rurale, en s'inquiétant de la tendance à l'abandon et, conséquemment, de la baisse en nombre et en qualité des médecins en milieu rural ${ }^{6}$, le problème persiste encore. Par ailleurs, les pouvoirs publics, convaincus à l'issue de la Première Guerre mondiale de l'importance d'une population en santé pour l'avenir d'une nation, mettaient en œuvre une kyrielle d'initiatives en vue de réduire la mortalité infantile. Au Canada et au Québec, les grands programmes gouvernementaux, inspirés par le mouvement de santé publique américain, s'étendent dès cette époque aux milieux ruraux et mettent l'accent sur l'éducation populaire.

Les mères en particulier, à qui les autorités politiques et médicales attribuent une part de responsabilités dans la mortalité infantile, deviennent la cible privilégiée d'interventions visant à leur inculquer des savoirs et des pratiques que l'on escompte les voir adopter ${ }^{7}$. La prise en charge de cette lutte par les pouvoirs publics n'élimine pas les nombreu-

5. L'historiographie consacrée à l'histoire de la colonisation, des services de santé, ou encore des infirmières reste pratiquement muette sur ce phénomène. Il existe quelques articles à caractère anecdotique mettant en relief le rôle joué par des infirmières de colonies dont ceux de Aurore Bégin publiés dans le Bulletin des gardes-malades catholiques, entre 1934 et 1941, et celui de Louis-Émile Hudon dans le Bulletin des infirmières catholiques du Canada, 12,6 (1945): 182-190. Des missionnaires et des infirmières ont également témoigné, parfois sous une forme romanesque, de leur expérience en colonies dans certaines régions du Québec. Voir en particulier Louis Garnier, eudiste, $D u$ cométique à l'avion. Les Pères Eudistes sur la Côte Nord (1903-1946) (Québec, A. D'Amours, C.J.M., 1947); et Bérith, Nicole Dionne de la Chevrotière, Rocabérant ou les tribulations d'une jeune infirmière chez les pionniers de l'Abitibi (Montréal, Éditions Sondec, 1974); également Marie Lefranc, La rivière solitaire (Paris, Ferenczi, 1934).

6. Cette question est largement documentée. Mentionnons l'intérêt plus récent pour des études de cas comme celle de Margaret W. Andrews, «Rural Medical Practice in the Smelter West, 1898-1923: a Case-Study Comparison», Canadian Bulletin of Medical History/Bulletin canadien d'histoire de la médecine, 6 (1989): 83-109. Celle-ci mentionne, à la page 80, que «From the 1920s, North American country doctors were increasingly considered underqualified - either as graying and hopelessly out of touch with recent medical developments or as quacks or alcoholics incapable of success in more promising settings.»

7. La propagande sanitaire à leur endroit s'immisce par tous les créneaux de communication disponibles: brochures, pamphlets, cinéma, radio, journaux locaux, annonces en chaire, etc. Katherine Arnup, «Educating Mothers: Government Advice for Women in the Inter-Wars Years», dans K. Arnup, A. Lévesque et R. R. Pierson, dir., Delivering Motherhood: Maternal Ideologies and Practices in the $19^{\text {th }}$ and $20^{\text {th }}$ Centuries (Londres et New York, Routhledge, 1990), 190-210. 
ses initiatives privées émanant souvent de groupes de femmes; elle modifie toutefois la nature des interventions premières: des médecins prendront la direction des activités et des infirmières remplaceront les bénévoles féminines ${ }^{8}$. La question des frontières interprofessionnelles se pose alors avec une acuité croissante. Si les femmes, premières concernées, semblent avoir joué un rôle actif dans le processus de médicalisation de la maternité, les infirmières qui jouent un rôle clé dans cette entreprise d'éducation en pénétrant dans les foyers, y contribueront indéniablement ${ }^{9}$. L'encadrement offert par les pouvoirs publics n'est pas sans failles et l'action des infirmières hygiénistes apparaît bien limitée dans les régions éloignées souvent dépourvues de tout autre ressource en matière de soins de santé. Comme l'ont bien montré C. CommachioAbeele et M. Stuart pour l'Ontario, les infirmières s'y trouvent placées en situation de conflit perpétuel entre les règles professionnelles leur interdisant de poser des diagnostics et d'effectuer des traitements et la nécessité, en l'absence de tout autre ressource médicale, d'assister les femmes en couche et de répondre aux urgences médicales ${ }^{10}$.

En France, selon O. Faure, l'encadrement sanitaire public se serait traduit, dès le XIX ${ }^{\mathrm{e}}$ siècle, par la volonté des législateurs de «soigner au meilleur coût le plus grand nombre possible de gens»; le choix des praticiens: médecins, infirmières, sages-femmes ou religieuses, correspondant davantage aux besoins et aux ressources de la population qu'à une

8. Les premières initiatives émanant de féministes anglophones, à qui l'on doit notamment les premiers centres de distribution de lait sain, et francophones, avec entre autres la création de l'hôpital Sainte-Justine pour enfants en 1907, sont ainsi modifiées par ces interventions, comme le montre bien Denyse Baillargeon, «Fréquenter les Gouttes de lait. L'expérience des mères montréalaises, 1910-1965», Revue d'histoire de l'Amérique française, 50,1 (été 1996): 29-68; et Aline Charles, Travail d'ombre et de lumière: le bénévolat féminin à l'Hôpital Sainte-Justine, 1907-1960 (Québec, Institut québécois de recherche sur la culture, 1990).

9. D. Baillargeon relève que les femmes des milieux populaires montréalais estimaient préférable le recours aux services des infirmières (de la Métropolitaine), jugées plus proches de leurs préoccupations, qu'à ceux des médecins (de l'Assistance maternelle), même dispensés gratuitement. Ces femmes ont également mentionné leur préférence pour les services à domicile plutôt qu'en clinique et jugé plus limitée la portée des seuls services de dépistage offerts par les Gouttes de lait. D. Baillargeon, «Care of Mothers and Infants in Montreal Between the Wars: the Visiting Nurses of Metropolitan Life, Les Gouttes de lait, and Assistance maternelle», Dianne Dobb et Deborah Gorham, dir., Caring and Curing. Historical Perspectives on Women and Healing in Canada (Ottawa, University of Ottawa Press, 1994), 163-181. Pour l'Ontario, C. Commachio-Abeele soutient que les infirmières étaient vues par les autorités publiques comme les «foot-soldiers of the crusade», «"The Mothers of the Land Must Suffer": Child and Maternal Welfare in Rural and Outpost Ontario», Ontario History, 80,3 (september 1988): 184.

10. C. Commacchio-Abeele, op. cit. et «Nations Are Built of Babies», Saving Ontario's Mothers and Children, 1900-1940 (Montréal et Kingston, McGill-Queen's University Press, 1993); Meryn Stuart, «Ideology and Experience: Public Health Nursing and the Ontario Rural Child Welfare Project, 1920-25», CBMH/BCHM, 6 (1989): 111-131. 
quelconque volonté de contrôle étatique ${ }^{11}$. Au Canada, le contrôle obtenu par la profession médicale sur l'ensemble du champ sanitaire et la volonté d'une élite d'infirmières de se tailler un statut professionnel enviable ont concouru à l'élimination progressive des sages-femmes ${ }^{12}$. L'origine d'un service infirmier au Québec appelé Service médical aux colons soulève plusieurs questions, notamment quant aux motifs du gouvernement, au concept de dispensaire-résidence, au choix des infirmières, à la durée et à la gratuité du service ou à son statut légal et administratif. Nous avons voulu rendre compte de l'organisation d'un tel service en situant le phénomène des dispensaires-résidences de colonies à l'intersection du développement du système de santé publique dans la province ${ }^{13}$ et de l'utilisation d'une catégorie particulière d'infirmières appelées infirmières de colonies.

Le Service médical aux colons (SMC) officiellement créé en 1936 pour superviser la mise en place, le financement et l'administration des dispensaires-résidences d'infirmières, devenait en 1943 l'une des divisions du ministère de la Santé du Québec. L'année suivante, le SMC supervisait un réseau de 113 postes d'infirmières pour l'ensemble de la

11. Olivier Faure, Les Français et leur médecine au XIXe siècle (Paris, Belin, 1993), 14.

12. Ces questions ont été bien documentées. Voir notamment Hélène Laforce, Histoire de la sage-femme dans la région de Québec (Québec, Institut québécois de recherche sur la culture, 1985); Jacques Bernier, La médecine au Québec. Naissance et évolution d'une profession (Québec, Les Presses de l'Université Laval, 1989); Suzann Buckley, «Ladies or Midwives? Efforts to Reduce Infant and Maternal Mortality», dans Linda Kealey, dir., A Not Unreasonable Claim. Women and Reform in Canada, 1880s-1920s (Toronto, The Women's Press, 1979), 131-149; Beverly Boutilier, «Helpers or Heroines? The National Council of Women, Nursing, and "Women's Work" in Late Victorian Canada», dans D. Dobb et D. Gorham, op. cit., 17-47; D. Baillargeon, «Les rapports médecins-infirmières et l'implication de la Métropolitaine dans la lutte contre la mortalité infantile, 1909-1953», The Canadian Historical Review, 77,1 (mars 1996): 33-60; Yolande Cohen et Louise Bienvenue, «Émergence de l'identité professionnelle chez les infirmières québécoises, 18901927», CBMH/BCHM, 11 (1994): 119-151. La question des frontières interprofessionnelles n'est pas étrangère aux transformations d'ensemble survenues dans la profession d'infirmières, comme le montre bien Kathryn McPherson, Bedside Matters. The Transformation of Canadian Nursing, 1900-1990 (Toronto/New York/Oxford, Oxford University Press, 1996), en particulier le chapitre 2, 26-73. Cette question se posera également avec acuité pour d'autres professions paramédicales largement féminisées, comme les physiothérapeutes et les diététistes dont traitent Nadia FahmyEid et al., Femmes, santé et professions (Montréal, Fides, 1997), en particulier le chapitre 10, 235261.

13. Pour une vue d'ensemble de ce développement, voir François Guérard, Histoire de la santé publique au Québec (Montréal, Boréal, coll. «Boréal express», 1996); Hervé Anctil et MarcAndré Bluteau, «La santé et l'assistance publique au Québec, 1886-1986», Santé et société (Édition spéciale, 1986). L'étude de G. Desrosiers, B. Gaumer, O. Keel, La santé publique au Québec. Histoire des unités sanitaires de comté, 1926-1975 (Montréal, Presses de l'Université de Montréal, 1998), démontre bien que les unités sanitaires, qui prennent en charge l'ensemble des activités publiques de prévention et d'hygiène du milieu, favorisent un quadrillage sanitaire de plus en plus serré. Ce réseau de santé touche déjà, en 1934, le tiers de la population estimée de la province. 
province ${ }^{14}$. La division du SMC fut abolie en 1962 et ses infirmières, jugées encore utiles, voire indispensables, intégrées alors à la division des unités sanitaires, elle-même abolie en 1972 à la suite des travaux de la commission Castonguay-Nepveu. Malgré la réforme du système de santé, les nouvelles structures sanitaires devaient maintenir en opération et, dans certains cas, ouvrir encore quelques dispensaires-résidences tenus par des infirmières ${ }^{15}$. Cet article porte sur la gestation et l'implantation de ce service infirmier de 1932 à 1943. Il vise à démontrer que le gouvernement du Québec, en dirigeant un mouvement de colonisation dans les régions éloignées de la province pendant la crise des années 1930, fut amené à mettre en place, à financer et à administrer un réseau de dispensaires-résidences dirigés par des infirmières, en dépit des politiques sociales et des normes professionnelles de l'époque.

Nous tenterons de démontrer comment ce service, présenté comme «la solution la plus raisonnable», a fini par s'imposer, malgré la réticence des autorités, et comment il permettait de répondre, de façon satisfaisante, à un ensemble de besoins. Ce qui expliquerait que le gouvernement a non seulement maintenu cette initiative bien au-delà de la crise économique mais encore il l'a étendue à travers la province. Appuyée principalement sur l'analyse des archives du SMC et sur ses rapports annuels d'activités diffusés par le ministère de la Santé de la province ${ }^{16}$, la reconstitution de l'histoire d'un service considéré comme un «cas d'espèce» au Québec nous a posé plusieurs difficultés dont nous avons traité ailleurs ${ }^{17}$.

14. SMC, Rapport annuel, 1944, Troisième rapport du ministère de la Santé, Québec, 12 janvier 1948, 269. Selon nos données, pas moins de 174 postes au total ont été créés de 1932 à 1975.

15. C'est le cas notamment d'Aylmer Sound, sur la Côte-Nord, ouvert en 1986.

16. Cet article s'appuie essentiellement sur les sources suivantes: le fonds Archives nationales du Québec (Québec)-Affaires sociales. Dispensaires. Service médical aux colons (ci-après nommé ANQ-SMC); le fonds Archives nationales du Québec-Affaires sociales. Unités sanitaires. Unité sanitaire Abitibi (ci-après nommé ANQ-US) et un fonds non classifié disponible aux Archives nationales du Québec-Région de l'Abitibi-Témiscamingue (ci-après nommé ANQ-AT). Le fonds ANQ-SMC compte 9 boîtes renfermant chacune plusieurs dossiers. Nous y référons dans cet article par les lettres $\mathrm{B}$ et $\mathrm{D}$ pour indiquer respectivement la boîte et le dossier concernés. Ce fonds renferme principalement de la correspondance échangée entre les directeurs successifs du service, les autorités des ministères de la Santé et de la Colonisation et les infirmières postées dans les dispensaires de l'ensemble de la province. Il compte aussi divers rapports, mémoires et directives administratives. Le fonds ANQ-US a permis de retracer des informations précises sur les origines du SMC. Quant au fonds ANQ-AT, il contient le même type d'informations que le fonds ANQ-SMC mais pour les dispensaires de l'Abitibi-Témiscamingue. Notons enfin que les rapports annuels du SMC, publiés de 1943 à 1971, ont constitué également une source majeure de renseignements.

17. Johanne Daigle, Nicole Rousseau et Francine Saillant, «Des traces sur la neige. La contribution des infirmières au développement des régions isolées du Québec au $\mathrm{XX}^{\mathrm{e}}$ siècle», Recherches féministes, 6,1 (1993): 93-103. 
Pour cette raison, nous avons dû exclure, à regret, l'étude des dispensaires gérés par le gouvernement fédéral auprès des populations amérindiennes.

\section{UNE SOLUTION INSPIRÉE PAR LA NÉCESSITÉ (1932-1935)}

La solution québécoise présente certaines similitudes avec celles adoptées précédemment dans d'autres provinces canadiennes. Ainsi, l'idée d'utiliser des infirmières spécialement formées en obstétrique pour soigner les femmes en couche émerge dans le contexte de la colonisation intensive des provinces des Prairies au début du $\mathrm{XX}^{\mathrm{e}}$ siècle. À l'initiative des groupes de femmes, en particulier des fermières de l'Alberta, les pouvoirs publics provinciaux autorisaient, en 1919, la création du Alberta District Nursing Service, mandaté pour donner des services médicaux d'urgence et s'occuper des femmes enceintes ${ }^{18}$. À Terre-Neuve, au début des années 1920, les autorités de santé locales avaient établi le Newfoundland Outpost Nursing and Industrial Association (NONIA) en amenant sur l'île les premières infirmières sages-femmes formées pour œuvrer dans les colonies isolées, la plupart recrutées des îles Britanniques ${ }^{19}$. La multiplication d'études de cas sur les services infirmiers, au Canada et ailleurs dans le monde, permettra éventuellement de mieux cerner la spécificité du cas québécois et d'établir des comparaisons ${ }^{20}$.

Au Québec, on dénombrait, avant la grande crise, quelques infirmières en poste dans des endroits éloignés et dépourvus de médecins ${ }^{21}$. Nos

18. Pour l'Alberta, voir Sharon Richardson, «Political Women, Professional Nurses and the Creation of Alberta's District Nursing Service, 1919-1925», Nursing History Review, 6 (1998): 2550; et Frontier Health Care: Alberta's District Nursing Service, 1919-1976, Communication présentée à la Conférence annuelle de la Société canadienne pour l'histoire de la médecine, Memorial University, St-John's, Newfoundland, 8 juin 1997. Nanci Langford dans «Childbirth on the Canadian Prairies, 1880-1930» a bien fait ressortir, à travers l'analyse des lettres et des journaux intimes de 78 femmes venues s'établir dans les provinces des Prairies entre 1880 et 1930, les conséquences souvent désastreuses de l'accouchement en l'absence de ressources sanitaires adéquates.

19. Cecilia Benoit, Midwives in Passage. The Modernisation of Maternity Care (St.John's, Newfoundland, Institute of Social and Economic Research, Memorial University of Newfoundland, 1991), 19. Le département de Santé avait pris soin, note l'auteure qui en trace un portrait plutôt négatif, de sélectionner les endroits où les infirmières auraient accès à un ministre du culte ou à un juge de paix dont la tâche était de voir à ce qu'elles ne soient pas surchargées. En 1933, après une décennie d'opération, le nombre de ces cliniques était passé de 25 à 8.

20. Les communications de Nancy Edgecombe de Yellowknife au Canada, «The Evolution of Nursing in the Northwest Territories» et de Jeanette A. Klotz de Rockhampton en Australie, «They Also Serve Who Stand and Wait - Remote Area Nurses at Birdsville, Australia, 1939-1945», toutes deux présentées sous les auspices de la Canadian Association for the History of Nursing, International History of Nursing Conference, Vancouver, 12-15 juin 1997, révèlent un intérêt croissant pour l'étude de services infirmiers dans les régions et localités dépourvues de médecins.

21. Il y en aurait eu une à Lamorandière en 1917 et une autre à Desmeloïze en 1921, deux localités ouvertes à la colonisation dans la région de l'Abitibi. 
sources ne nous permettent pas de connaître les conditions entourant la création de ces postes, mais l'ouvrage de Louis Garnier, missionnaire eudiste, nous en donne un aperçu. Dans son récit relatant l'expérience des missionnaires eudistes sur la Côte-Nord dans les premières décennies du $\mathrm{XX}^{\mathrm{e}}$ siècle, il rapporte qu'en l'absence de médecins, «Presque tous [les missionnaires] s'occupaient de médecine, donnaient des conseils, proposaient des remèdes connus. Plusieurs étaient devenus experts dans l'extraction des dents. [...] Mais combien de fois sont-ils sortis de la chambre des malades perplexes, soucieux, ne sachant que faire ni que dire $^{22}$ !» Il faut rappeler que l'Église catholique, le plus souvent par le biais des congrégations féminines, a généralement considéré les soins de santé comme partie intégrante de ses œuvres missionnaires, comme en témoignent l'exemple des sœurs Grises et celui des sœurs de Sainte-Anne ${ }^{23}$. En 1922, le père Garnier se serait adressé au docteur Alphonse Lessard, afin d'obtenir des médecins pour desservir les centres les plus importants de la région. La double position occupée par ce médecin, directeur du Service provincial d'hygiène $(\mathrm{SPH})$ et du Service de l'assistance publique (SAP), deux services nouvellement créés $^{24}$, lui conférait une vision d'ensemble aussi bien des besoins des populations que des contraintes budgétaires ${ }^{25}$.

À la suite des pressions faites par le missionnaire Garnier en poste à Rivière-au-Tonnerre, une petite localité de la Basse-Côte-Nord, le docteur Lessard commandait une inspection médicale de cette région dont le rapport, déposé en 1924, faisait état de conditions hygiéniques

22. Le père Louis Garnier, eudiste, $o p$. cit., 185.

23. Pauline Paul, «The Contribution of Grey Nuns to the Development of Nursing in Canada: Historiographical Issues», CBHM/BCHM, 11 (1994): 207-217; Sister Margaret Cantwell, S.S.A., North to Share. The Sisters of Saint Ann in Alaska and the Yukon Territory (Victoria, Sister of Saint Ann, 1997). Pour une vue d'ensemble de l'ampleur du travail des religieuses hospitalières, voir Nicole Laurin, Danielle Juteau et Lorraine Duchesne, À la recherche d'un monde oublié. Les communautés religieuses de femmes au Québec de 1900 à 1970 (Montréal, Le Jour, 1991); et plus récemment des deux premières auteures, Un métier et une vocation. Le travail des religieuses au Québec de 1901 à 1971 (Montréal, Presses de l'Université de Montréal, 1997).

24. Selon G. Desrosiers, B. Gaumer et O. Keel, op. cit., le SPH s'occupait aussi d'administrer l'assistance publique tout en poursuivant l'œuvre de promotion de l'hygiène amorcée par le Conseil d'hygiène. Le SPH, relevant directement du Secrétariat de la province, pouvait ainsi pousser plus loin ses interventions. Voir F. Guérard, op. cit., en particulier le chapitre 4, 45-59. Le docteur Lessard fut directeur des deux services de 1922 à 1936. Dans la correspondance échangée à propos du SMC pour les années 1935 et 1936, ce dernier s'identifie tantôt comme directeur de l'un, tantôt comme directeur de l'autre service.

25. Le docteur Lessard avait d'abord pratiqué la médecine générale à Rivière-du-Loup, une petite ville de l'est de la province, puis enseigné à la Faculté de médecine de l'Université Laval avant d'être nommé à la direction du SPH et du SAP. En 1925, il se rendra aux États-Unis à l'invitation de la fondation Rockefeller et implantera les premières unités sanitaires de comté du Québec à partir de l'année suivante. 
«lamentables» dans les 17 municipalités visitées entre Godbout et Natashquan $^{26}$. En 1926, le directeur du SPH autorisait l'embauche d'une infirmière; il s'agit du premier poste pour lequel nous possédons quelques informations. C'est ainsi que le 26 août 1926, l'infirmière Éveline Bignell s'installait dans une maison louée pour y recevoir et y soigner toute personne s'y présentant, pratiquer des accouchements et faire des visites à domicile au besoin. Deux autres infirmières étaient embauchées de la même façon en 1929 pour desservir Rivière-Saint-Jean et Natashquan. L'idée de recourir aux services des infirmières se serait imposée d'emblée, selon le missionnaire, compte tenu des sommes élevées exigées par des médecins pour desservir ces localités pauvres: «Voilà donc [concluait-il] qu'un problème jadis source d'angoisse, a reçu sa solution la plus raisonnable pour la Côte-Nord et pour les localités pauvres et isolées ${ }^{27}$.»

En 1932, le docteur Lessard envisageait d'adopter cette solution lors de l'ouverture de nouveaux centres de colonisation de la province ${ }^{28}$. Il lui faudra des années pour la faire accepter et financer sur une base régulière, sinon permanente.

\section{«La solution la plus raisonnable» tolérée sur une base temporaire}

Au printemps de 1932, devant l'ampleur de la crise économique, le gouvernement fédéral adoptait le plan Gordon de «retour à la terre» comme mesure de secours aux chômeurs des villes, auquel devaient contribuer financièrement les gouvernements provinciaux et municipaux pour l'établissement de familles sur des terres. L'allocation offerte (600\$) s'était avérée insuffisante, si bien que le gouvernement du Québec avait dû soutenir financièrement les colonies naissantes en payant les primes de défrichement, la construction d'écoles, le salaire des fonctionnaires, des institutrices et celui des infirmières dépêchées dans les

S'adjoignant le docteur Émile Nadeau, qui avait acquis son expérience dans les faubourgs de Québec, il aura conféré au développement des services de santé publique une impulsion décisive. Le gouvernement de l'Union nationale, porté au pouvoir en août 1936, l'aurait enjoint à prendre sa retraite. Le ministère de la Santé nouvellement créé allait être dirigé temporairement par le docteur Nadeau et, à compter de décembre 1937, par le docteur Jean Grégoire, médecin hygiéniste nommé sous-ministre, poste qu'il conservera jusqu'en 1962. Ces informations sont tirées de G. Desrosiers, B. Gaumer et O. Keel, op. cit., chapitre 2, 13-47.

26. ANQ (SMC), B1-D3, Rapport d'inspection médicale, transmis au docteur Alphonse Lessard, directeur du SPH, le 5 septembre 1924, par le docteur L. P. Savoie, après un séjour de deux mois sur la Côte-Nord.

27. Le père Louis Garnier, op. cit., 193.

28. ANQ (SMC), B4-D1, Alphonse Lessard, directeur du SPH, à Hector Laferté, ministre de la Colonisation, Québec, 29 décembre 1932. 
endroits où les besoins apparaissaient urgents ${ }^{29}$. Dès les premiers essais, l'envoi d'infirmières plutôt que de médecins, même comme solution temporaire, avait rencontré quelques obstacles. Le poste consenti à la colonie d'Auclair (Bas-Saint-Laurent) l'illustre bien. Le docteur Lessard écrivait au ministre de la Colonisation, en décembre 1932, qu'il avait déjà reçu «des protestations de la part de certains médecins qui s'opposent à la nomination d'infirmière ${ }^{30}{ }^{30}$. En janvier 1933, dans sa lettre d'embauche de l'infirmière d'Auclair, Gabrielle Blais, le docteur Nadeau lui exposait ainsi la situation en précisant le caractère temporaire de son poste:

[...] comme la chose vous a été expliquée, jusqu'à l'établissement d'un médecin dans le territoire qui vous a été assigné, vous aurez la latitude de donner les soins médicaux requis à la population, faire les accouchements et bien suivre le cas de chaque malade, de façon à fournir un service médical aussi complet que possible. Cependant, s'il survient un cas spécial d'une gravité exceptionnelle, il vous faudra user de votre bon jugement et vous entendre avec les intéressés ou le curé de l'endroit pour qu'un médecin soit appelé; mais dans ces cas, le Service de l'Assistance publique ne sera pas responsable pour les frais encourus, à moins d'une entente spéciale pour chaque $\operatorname{cas}^{31} \ldots$

Les directives adressées aux infirmières indiquent bien le souci du SPH de diminuer les risques de conflits potentiels avec les médecins ou les politiciens locaux ${ }^{32}$. Au début des années 1930, quelques autres infirmières étaient nommées, pour les mêmes raisons d'économie et toujours sur une base temporaire, en Abitibi-Témiscamingue (Aurore Bégin en 1932 à Rollet, Blanche Esnouf en 1934 à Sainte-Gertrude de Manneville, Murielle Lemieux à Lac Barrière en 1935), au Saguenay (Anita Dionne en 1932 à L'Anse Saint-Jean) et dans le Bas-Saint-Laurent (Luce Thibault le $1^{\mathrm{er}}$ janvier 1932 à Biencourt); il ne s'agit ici que des infirmières pour lesquelles nos sources nous permettent de préciser l'année d'embauche.

29. Roger Barette, «Le plan Vautrin et l'Abitibi-Témiscamingue», dans Maurice Asselin et Benoît Beaudry-Gourd, dir., op. cit., 105-110; Michel Pelletier et Yves Vaillancourt, op. cit., 247266; et Esdras Minville, op. cit.

30. ANQ (SMC), B4-D1, Alphonse Lessard à Hector Laferté, Québec, 29 décembre 1932.

31. ANQ (SMC), B4-D1, Émile Nadeau, directeur-adjoint du SPH, à Garde Blais, infirmière d'Auclair, Québec, 14 janvier 1933.

32. L'étude de G. Desrosiers, B. Gaumer et O. Keel, déjà citée, confirme ce souci du docteur Lessard dans la mise sur pied des unités sanitaires de comté. 
Pressé de jouer un rôle plus actif dans le mouvement de colonisation et d'agir en faveur de ce que plusieurs considéraient comme «une œuvre de restauration nationale ${ }^{33}{ }$, le gouvernement libéral de L.-A. Taschereau annonçait, le 8 août 1934, son intention de soumettre à la Législature un plan de colonisation d'ensemble (devenu le plan Vautrin), entièrement conçu et financé par la province ${ }^{34}$. La crise économique ravivait en effet le nationalisme canadien-français en remettant à l'ordre du jour, devant l'échec perçu des politiques économiques libérales, la traditionnelle vision agricole, familiale et catholique des Canadiens français ${ }^{35}$. Dans ce contexte, lors du Congrès de la colonisation tenu à Québec les 17 et 18 octobre 1934, auquel le gouvernement libéral avait convoqué tout spécialement les évêques et les missionnaires colonisateurs pour discuter des modalités d'application du plan projeté, le docteur Lessard informait les quelque trois cents congressistes réunis que son service (le SPH) avait établi des infirmières dans les localités nommées précédemment pour assurer aux colons un minimum d'assistance médicale. Il insistait encore une fois sur le caractère extraordinaire et temporaire de ces mesures: «Nous subissons une crise, et dans les temps extraordinaires il s'agit de prendre les moyens extraordinaires.» Il ajoutait: «À part cela, nous avons un médecin qui circule constamment d'une extrémité de la Province à l'autre [...]. Il surveille le travail des infirmières. Quand il passe dans les régions de colonisation, il donne des consultations ${ }^{36} . »$

Le médecin circulant à travers la province était le docteur Émile Martel, devenu médecin-hygiéniste régional et officier médical de l'unité sanitaire du comté d'Abitibi établie en 1934. Les congressistes refusèrent cependant de sanctionner la solution proposée par le docteur Lessard qui consistait à nommer «un médecin dans une région avec, sous ses ordres, un certain nombre d'infirmières»; le directeur du SPH leur avait pourtant avoué que «[...] dans toutes les régions de colonisation où nous avons développé un système, l'effet a été magnifique ${ }^{37}$.» Ils appuyèrent plutôt une proposition présentée par le docteur J.-E.

33. Plusieurs auteurs font état des débats entourant cette question dont Raoul Blanchard, Roger Barette, Michel Pelletier et Yves Vaillancourt, déjà cités. Les Actes du Congrès de la colonisation tenu à Québec les 17 et 18 octobre 1934 sous la présidence de l'honorable Irenée Vautrin, ministre de la Colonisation (Québec, 1935), 266 p., donnent par ailleurs un bon aperçu des diverses facettes du projet soumis par le nouveau ministre de la Colonisation, Irenée Vautrin.

34. Le Devoir, 9 août 1934.

35. Sur les débats idéologiques pendant la crise, voir Fernande Roy, Histoire des idéologies au Québec aux XIX et XX $X^{e}$ siècles (Montréal, Boréal, coll. «Boréal express», 1993), 83-92.

36. Actes du Congrès de la colonisation tenu à Québec..., 215-216.

37. Ibidem, 216. 
Desroches, président des sociétés médicales de colonisation, voulant que, dans son programme de colonisation, le gouvernement provincial «voit à la nomination de médecins [...] au lieu de payer les services d'une garde-malade ${ }^{38}{ }_{\gg} \ldots$ Cependant, aucune des lois relatives à la colonisation sanctionnées pendant les années 1930 n'obligeait le gouvernement provincial à assurer un tel service médical; la résolution du docteur Desroches restera lettre morte. On ne connaît toutefois pas la nature, l'étendue, ni le poids politique des sociétés médicales de colonisation; la recherche reste encore à faire sur ce point.

Avant que les budgets spéciaux soient votés pour la mise en œuvre du plan Vautrin, le docteur Martel avait tenté de faire assumer par le SPH les coûts de l'embauche d'un médecin en proposant au docteur Lessard la mise à l'essai, dans la localité de Duparquet en Abitibi, d'un système de «secours médicaux aux colons» consistant à accorder une subvention annuelle à un médecin déjà à l'emploi d'une compagnie minière en échange de ses services médicaux aux colons ${ }^{39}$. Le docteur Lessard lui répondit: «[...] je crois que nous pourrons faire un arrangement définitif avec le ministère de la Colonisation dès que la session de la législature sera terminée et que les crédits spéciaux auront été votés ${ }^{40}$.» Un budget total de $10000000 \$$ fut finalement voté en avril 1935 pour subventionner, à divers degrés, l'établissement et le maintien de colons sur des terres ${ }^{41}$; le gouvernement escomptait, qu'au bout de quelques années, ces familles subviendraient à leurs besoins.

Le ministère de la Colonisation devenait un ministère autonome et, en octobre de la même année, les services médicaux aux colons relevaient de ce ministère plutôt que du SPH. Dans une lettre circulaire adressée aux chefs de districts sanitaires de la province, J.-E. Caron, directeur des Services, écrivait: «Notre Département [la Colonisation] paiera désormais les comptes des médecins qui soigneront nos colons nécessiteux, dans les colonies où il n'y a pas de service médical organisé $^{42} . »$ Cette prescription était toutefois assortie de conditions très

\footnotetext{
38. Ibidem, 241-242. 1935.

39. ANQ (US), B1-D1, Émile Martel, officier-médical, à Alphonse Lessard, Amos, 18 février

40. ANQ (US), B1-D1, Alphonse Lessard à Émile Martel, Québec, 23 février 1935.

41. Michel Pelletier et Yves Vaillancourt, op. cit., 253-255.

42. ANQ (US), B1-D1, J.-E. Caron, directeur des Services, ministère de la Colonisation, Lettre circulaire aux chefs de district [sanitaire], Québec, 28 octobre 1935. À la suite de cette directive, le docteur Lessard précise au docteur Martel que: «[...] toute cette question du service médical de ces nouvelles colonies relève du ministère de la Colonisation et nous agissons seulement comme aviseurs [sic].» ANQ (US), B1-D1, Alphonse Lessard à Émile Martel, officier médical, Québec, 29 octobre 1935 .
} 
limitatives: la visite du médecin recommandée au préalable par le chef du district sanitaire qui devait apposer ses initiales sur toutes les factures des médecins, lesquelles factures devaient finalement être approuvées par le SPH avant leur paiement. Et ces paiements ne valaient que pour les colons nécessiteux. «Nous ne pouvons évidemment étendre de telles dépenses aux cultivateurs ${ }^{43}{ }$, d'ajouter J.-E. Caron. Le plan projeté, bien loin de la solution que le docteur Martel avait suggérée, s'est révélé pratiquement impossible à appliquer dans les régions de colonisation considérant les distances à parcourir, le degré d'urgence de certains cas et la nécessité d'établir une distinction entre les colons indigents et les cultivateurs souvent pauvres. Les budgets affectés aux «secours médicaux des colons» restant très limités, les médecins continuèrent de bouder ces régions.

Par conséquent, en décembre 1935, on commençait à envisager sérieusement une alternative; l'intensité de la correspondance échangée entre les principaux acteurs durant ce mois donne un aperçu des pressions exercées. Le 4 décembre, le docteur Martel exprimait au docteur Lessard son désir de «discuter avec les autorités compétentes cette question de plus en plus importante» de «l'organisation du Service médical aux Colons», un sujet sur lequel, assurait-il, il possédait «un dossier assez complet ${ }^{44} \gg$. On sait, à travers un échange de cinq lettres ${ }^{45}$ entre les deux hommes, que la balle était dans le camp du ministère de la Colonisation, alors qu'une rencontre attendue était effectivement tenue à Québec, le 24 décembre 1935. Elle mettait en présence le directeur du SPH, les autorités du ministère de la Colonisation et le docteur Martel pour discuter de son projet. Ce projet, que son supérieur qualifiait de «document très bien préparé», est malheureusement absent des archives; nous ne savons donc pas s'il ressemblait à la solution proposée pour Duparquet. La suite des événements montre cependant que c'est «la solution la plus raisonnable» qui l'a emporté en cette veille de Noël 1935. Nous n'avons pas trouvé de compte rendu de cette réunion ni de

43. ANQ (US), B1-D1, J.-E. Caron, op. cit.

44. ANQ (US), B1-D1, Émile Martel à Alphonse Lessard, Amos, 4 décembre 1935.

45. ANQ (US), B1-D1, Alphonse Lessard à Émile Martel, officier médical, Unité sanitaire du comté de l'Abitibi, Québec, le 11 décembre 1935; ANQ (US), B1-D1, Émile Martel à Alphonse Lessard, Amos, le 13 décembre 1935; ANQ (US), B1-D1, Alphonse Lessard à Émile Martel, Québec, le 17 décembre 1935; ANQ (US), B1-D1, Alphonse Lessard à Émile Martel, Québec, 18 décembre 1935. Il y a deux lettres de Lessard à Martel datée du 18 décembre; dans l'une d'elles, il invite le docteur Martel à venir à Québec le 24 décembre pour discuter du projet. Dans l'autre, il lui confie: «Comme le ministère de la Colonisation est le décideur, on pourrait chercher des informations complémentaires du côté de ses représentants. Je pense à Ivanhoë Caron, prêtre colonisateur, organisateur, entre autres, du service d'assistance aux colons au sein de ce ministère entre 1911 et 1923 .» 
document officiel y faisant référence. Dans un dédale de dispositions législatives, nous avons cherché en vain une loi instituant le SMC. La création à titre expérimental des premières unités sanitaires de comté en 1926 n'avait pas non plus fait l'objet d'un arrêté en conseil.

Nous en sommes donc réduites à tenter de reconstituer le scénario le plus probable qui a conduit à la mise sur pied du SMC et permis d'expliquer son statut particulier et son fonctionnement dans la structure des services sociosanitaires de l'époque.

\section{Quand la nécessité impose sa loi...}

La réunion du 24 décembre 1935 paraît cruciale. Elle met en présence des acteurs gouvernementaux qui cherchent une solution et un autre, le docteur Martel, qui décrit la réalité quotidienne des services de santé en colonies. Le problème pouvait paraître insoluble pour les raisons suivantes: la seule disposition législative (le plan Vautrin) autorisant une forme de financement pour des services médicaux ne prévoyait qu'une solution impraticable; les problèmes de santé ne constituaient pas une priorité du ministère de la Colonisation; les médecins refusaient de s'établir en colonies; le SPH préconisait une solution inacceptable aux yeux des sociétés médicales de colonisation et n'avait pas les budgets pour engager des infirmières. Il fallait pourtant trouver un compromis qui puisse répondre aux besoins tout en s'accommodant des lois existantes et ce, en période de crise économique.

On peut imaginer les arguments invoqués en faveur de «la solution la plus raisonnable», certains de ces arguments ayant été plaidés par le docteur Lessard au congrès de 1934. Rappelons que la double fonction assumée par ce médecin, dirigeant à la fois le SAP et le SPH, lui conférait une position avantageuse pour faire accepter son projet. L'application normale de la Loi de l'assistance publique ne permettait pas de payer les frais d'hospitalisation des indigents résidant dans des territoires non organisés puisqu'elle prévoyait un financement tripartite impliquant la province, la municipalité de résidence du malade et l'hôpital concerné. Or, comme le soulignait le docteur Lessard, les colonies n'étaient pas constituées en municipalités organisées avec pouvoir de prélever une taxe (le sou du pauvre) destinée à financer le tiers du coût des hospitalisations.

Le problème s'était posé lors de l'établissement des premiers contingents de colons établis au Québec en vertu du plan Gordon; plusieurs de ces colons étaient malades et avaient été hospitalisés aux frais du $\mathrm{SAP}^{46}$.

46. Actes du Congrès de la Colonisation tenu à Québec..., 215. 
Le ministère de la Colonisation avait d'abord consenti à ce que les aspirants-colons subissent un examen médical avant d'être sélectionnés pour les colonies, une mesure essentielle mais insuffisante car, comme le soulignait le docteur Lessard, il fallait prévoir les cas de maternité, d'accidents et d'épidémies ${ }^{47}$. Or, rien dans la Loi de l'assistance publique ne permettait d'assurer les services médicaux, un argument contre l'octroi d'allocations à des médecins. L'article 16 était clair: «L'aide accordée par le gouvernement ne peut, dans aucun cas, dépasser le tiers du coût total de l'entretien des indigents recueillis par une institution d'assistance publique $^{48}$.» La loi laissait cependant au gouvernement la marge de manœuvre suivante: «Le lieutenant-gouverneur en conseil peut, néanmoins, sur recommandation du service de l'assistance publique, dans les cas d'urgence et de nécessité absolue, aider de la façon qu'il le juge à propos au développement des œuvres d'assistance publique de la province $^{49}$.»

Il restait à trouver la façon la plus «à propos» de répondre aux besoins urgents des colons en matière de services de santé. Ici, «la solution la plus raisonnable» acquiert toute sa pertinence, car les services d'infirmières, contrairement à ceux de médecins, pouvaient être considérés comme des services d'assistance minimale à des indigents. On peut supposer que l'idée d'établir ces infirmières dans des dispensaires-résidences s'inspire, d'une part, du concept des dispensaires apparus dans les villes à partir du $\mathrm{XIX}^{\mathrm{e}}$ siècle pour offrir des services médicaux spécialisés aux indigents et, d'autre part, des précédents établis au Québec, comme ailleurs au Canada, dans une utilisation plus importante des services des infirmières pour les régions dépourvues de tous services médicaux ${ }^{50}$. La résidence sur les lieux de travail, une pratique encore courante dans les milieux infirmiers de la première moitié du $\mathrm{XX}^{\mathrm{e}}$ siècle, permettait de résoudre le problème majeur de l'isolement géographique.

47. Ibidem, 214.

48. Loi établissant le Service de l'assistance publique, Statuts refondus de la Province de Québec, 1925, vol. III, 1925, Chapitre 189, articles 14, 15 et 16. Voir aussi Michel Pelletier et Yves Vaillancourt, op. cit., 98 et ss. Il faut préciser que le SAP est constamment déficitaire au cours des années 1930, comme le rappelle Gonzalve Poulin dans L'évolution de l'assistance au Québec, 16081951 (Québec, Éditeur officiel, 1955), 204 p., Annexe II du Rapport de la Commission royale d'enquête sur les problèmes constitutionnels (Commission Tremblay); et Serge Mongeau, Évolution de l'assistance au Québec (Montréal, Le Jour, 1967), 123 p.

49. Loi établissant le Service de l'assistance publique, op. cit., article 17.

50. Pour les dispensaires établis au Québec au XIX ${ }^{\mathrm{e}}$ siècle, voir Denis Goulet et André Paradis, Trois siècles d'histoire médicale au Québec. Chronologie des institutions et des pratiques (16391939) (Montréal, VLB éditeur, 1992). En ce qui concerne les dispensaires supportés par le SPH, voir F. Guérard, op. cit., 48-49. 
Un autre argument en faveur de l'ouverture de dispensaires pour y établir des infirmières pouvait être invoqué: la pauvreté. Le SPH avait reconnu, dès sa création en 1922, certains dispensaires spécialisés comme institutions d'assistance pour des clientèles cibles des villes populeuses, principalement les mères et les enfants. Il appliquait ainsi la directive de la Croix-Rouge à l'effet que «[...] les dispensaires sont largement ouverts non seulement aux indigents, mais d'une manière générale aux personnes n'ayant que de très modiques ressources ${ }^{51}$.» L'un des buts recherchés était alors de diminuer les coûts d'hospitalisation des indigents. Mais comment justifier la généralisation de telles institutions aux territoires de colonisation? Nous croyons que c'est en s'engageant à financer des postes d'infirmière dans les colonies, que le docteur Lessard, à titre de directeur du SAP, a réussi à persuader les représentants du ministère de la Colonisation de financer, du moins jusqu'à la création du ministère de la Santé et du Bien-être social ${ }^{52}$, la construction et l'entretien des dispensaires-résidences. Le principe d'un partage des coûts entre la province et les organismes locaux, enchâssé dans la Loi de l'assistance publi$q u e$, participe des valeurs et des pratiques traditionnelles de l'assistance encore fortement répandues avant la Deuxième Guerre mondiale ${ }^{53}$; le ministère de la Colonisation agissait en lieu et place d'une municipalité dans les territoires non organisés de la province.

Il restait encore à trouver une manière d'encadrer ces infirmières sur le plan administratif et juridique; un dernier argument pouvait être avancé. On pouvait s'appuyer sur certaines dispositions de la Loi créant le Service provincial d'hygiène en 1922 et de la Loi des unités sanitaires. La première loi prévoyait la possibilité de nommer, pour les territoires non organisés, des officiers d'hygiène, tout en laissant au titulaire le soin de définir leurs pouvoirs ${ }^{54}$. En vertu de cette disposition, le docteur Martel avait été nommé officier médical chargé de la surveillance des infir-

51. Rapport présenté par A. H. Deloges, directeur, et J. A. Ranger, assistant-directeur, Division des maladies vénériennes, reproduit dans le Rapport du secrétaire et du registraire de la province de Québec pour l'année 1921-1922 (Québec, Imprimeur du Roi, 1922), 106. Santé.

52. Précisons que le SMC a été mis sur pied neuf mois avant la création du ministère de la

53. Les nombreux ouvrages sur l'histoire de l'assistance publique, qu'il serait trop long de citer ici, en témoignent éloquemment. Mentionnons par ailleurs que les exemples de services infirmiers cités précédemment pour l'Alberta et Terre-Neuve s'appuient sur ce principe de partage des coûts: aux personnes assistées revenait la responsabilité des coûts de transport et de certains frais d'entretien de l'infirmière en service, comme le chauffage par exemple.

54. Loi concernant les unités sanitaires (Québec, Imprimeur du Roi, 1941), article 3, Chapitre 183; Statuts refondus de la province de Québec, 1941; Loi créant le Service provincial d'hygiène, article 138. 
mières dans les régions de colonisation en 1933, année où la Loi des unités sanitaires assurait la permanence de ces organisations ${ }^{55}$. Les dispensaires-résidences de colonies pouvaient ainsi être placés soit sous l'autorité de l'officier assigné aux territoires non organisés (ce dernier deviendra directeur du SMC), soit sous celle de l'officier médical d'une unité sanitaire de comté. Dans un cas comme dans l'autre, le SMC pouvait être mis sur pied, même s'il n'était assuré en vertu d'aucune loi relative à la santé publique.

Nous pensons que c'est essentiellement sur ces dispositions inscrites en marge des trois lois citées que se sont appuyés les protagonistes du SMC pour établir et maintenir ce service. Il est évident qu'on n'a pas voulu invoquer ces dispositions pour rémunérer des médecins, jugeant sans doute que les sommes réclamées par ceux-ci étaient trop élevées dans un contexte de crise et sachant fort bien qu'ils ne voulaient pas «s'exiler» dans ces régions. Les points de vue de deux autres auteurs nous encouragent dans cette interprétation des faits connus ${ }^{56}$. La réunion du 24 décembre 1935 n'a pas permis d'éliminer tous les obstacles à «la solution la plus raisonnable»; elle en a seulement autorisé une implantation discrète.

\section{L'IMPLANTATION DU SMC: DE L'IMPROVISATION À LA RECONNAISSANCE OFFICIELLE (1936-1943)}

Dès janvier 1936, les autorités concernées reconnaissaient que les services de santé en colonies devaient être confiés à des infirmières plutôt qu'à des médecins. On ne savait pas encore comment le système fonctionnerait, c'est-à-dire comment les trois instances concernées (le SPH, le SAP et le ministère de la Colonisation) se partageraient les responsabilités de financement et de gestion de ce service. De l'improvisation est née une structure qui commença à s'organiser à la veille de la création du ministère de la Santé et du Bien-être social du Québec en novembre 1936. Après sept ans de rodage, le SMC devenait, nous l'avons déjà précisé, une division officielle du ministère de la Santé avec ses propres règlements. pitre 184

55. Statuts refondus de la province de Québec, 1941 (Québec, Imprimeur du Roi, 1941), Cha-

56. Claire Martin, L'infirmière de colonie en Abitibi-Témiscamingue, récit historique présenté par la Corporation «Le dispensaire de la garde», La Corne, Abitibi, au ministère des Affaires culturelles du Québec, Direction de l'Abitibi-Témiscamingue, Programme d'aide aux organismes en matière de patrimoine, automne 1992; François-Albert Angers, op. cit. 


\section{Une improvisation explicable}

Ces tâtonnements administratifs, parfois exaspérants pour les infirmières, peuvent s'expliquer par une certaine négligence, on le verra, du ministère de la Colonisation, par un espoir tenace de recruter des médecins et par l'obligation constante de démontrer l'urgence et l'importance des besoins pour obtenir les budgets nécessaires. L'année 1936 en fut une de négociations entre les instances concernées. Théoriquement, le SAP prenait en charge le salaire des infirmières de même que les allocations aux médecins chargés de leur porter assistance au besoin, alors que le ministère de la Colonisation était chargé de la construction et de l'entretien des dispensaires, des moyens de transport et des autres conditions matérielles à régler sur le terrain. Mais la situation restait encore ambiguë, comme on peut le constater dans la lettre d'embauche adressée à Jeanne Chabot par le docteur Lessard le 13 janvier 1936:

[...] il a maintenant été décidé que vous serez au Service de l'Assistance publique, à partir du 15 décembre 1935, au traitement de $\$ 100.00$ par mois. Il est entendu qu'il s'agit d'une position temporaire pour le temps qu'il nous faudra maintenir un service médical gratuit pour les colons, pourvu que vos services soient trouvés satisfaisants. Dès que nous aurons terminé nos négociations avec le Ministère de la Colonisation, je vous écrirai de nouveau pour vous énumérer toutes les conditions de votre engagement et vous donner les instructions nécessaires. Vous serez alors sous la direction immédiate du Dr Émile Martel, officier médical de notre Unité sanitaire du comté de l'Abitibi, lequel sera chargé de la direction générale du service médical aux colons de l'Abitibi et du Témiscamingue ${ }^{57} \ldots$

La lettre d'embauche de Marguerite Turgeon en septembre 1936 est plus explicite quant au partage des responsabilités entre le SAP et le ministère de la Colonisation: «[...] vous devrez voir vous-même à vos frais de pension. Les questions de logement, ameublement, entretien, chauffage, éclairage et transport seront réglées par le Ministère de la Colonisation ${ }^{58}$.» À quelques reprises, plusieurs infirmières, de même que les autorités du SPH se plaindront du fait que le ministère de la Colonisation s'acquitte mal de ses engagements. À titre d'exemple, Aurore Bégin, l'infirmière en poste à Rollet (Rivière Solitaire) depuis 1932, habitait encore, en octobre 1936,

57. ANQ (AT), Alphonse Lessard, directeur du SAP, à garde Jeanne Chabot, infirmière de Sainte-Gertrude, Québec, 13 janvier 1936. Cette infirmière faisait parvenir à son supérieur son premier rapport hebdomadaire d'activités en date du 18 décembre 1935.

58. ANQ (AT), Alphonse Lessard, directeur du SAP, à Marguerite Turgeon, Québec, 18 septembre 1936. Notons que les colons devaient, en principe, approvisionner eux-mêmes l'infirmière en bois de chauffage. 
une «cabane» qui «ne mérite pas le nom de dispensaire», selon un rapport du ministère de la Santé; elle devait puiser son eau «au sceau [sic] à la rivière $^{59}$ !», et l'infirmière Patry écrivait, pour la quatrième fois dans l'année au docteur Lessard, le 8 mai 1936, pour qu'on lui fournisse un cheval ${ }^{60}$.

Ces négligences peuvent s'expliquer en partie par le fait qu'on a longtemps entretenu l'espoir d'attirer des médecins, d'où le manque d'empressement à investir dans des installations permanentes décentes pour les infirmières. La population semblait pourtant satisfaite des services des infirmières, si l'on en croit le témoignage du député de l'Abitibi, Émile Lesage: «Je tiens à vous dire que d'après les correspondances que j'ai échangées depuis quelques mois avec les colons de différents endroits de mon comté, ces derniers préfèrent de beaucoup des gardes-malades aux médecins ${ }^{61} . \gg$ Après quelques vaines tentatives pour obtenir les services de médecins dans les colonies, le docteur Nadeau, directeur intérimaire du ministère de la Santé, paraissait y avoir renoncé, en février 1937. Dans sa réponse au curé de Beaudry, il avouait: «Vous savez que l'honorable ministre de la Santé a fait un appel pressant aux médecins pour qu'ils offrent leurs services. Je dois vous dire que les demandes sont plutôt rares, et sont encore plus rares les demandes venant de médecins compétents, sobres, honnêtes et consciencieux ${ }^{62}$.» Il devait s'accommoder lui aussi de «la solution la plus raisonnable». Le SMC devait demeurer un service infirmier.

En mai 1936, le SMC, logé dans les bureaux de l'unité sanitaire de l'Abitibi à Amos, disposait d'un budget séparé de celle-ci ${ }^{63}$. Relevant de la division de l'Assistance publique, rattachée directement au secrétariat

59. ANQ (SMC), B4-D5, Rapport anonyme et non daté du Ministère de la Santé; également, ANQ (SMC) B4-D5, Rapport concernant le dispensaire de Rollet, du docteur Martel, le 14 octobre 1936.

60. ANQ (SMC), B2-D2, Marguerite Patry à Alphonse Lessard, Bellecombe, 8 mai 1936. L'examen des rapports du ministère de la Colonisation nous a permis de constater qu'ils sont à toutes fins utiles muets sur le SMC et sur les infirmières de colonies. À la décharge de ce ministère, il faut préciser que le subside total annuel qui lui était consenti est passé de 5657340 en 1939 à $671479 \$$ en 1944.

61. ANQ (SMC), B2-D8, Émile Lesage, député de l'Abitibi, à l'hon. H.-L. Auger, ministre de la Colonisation, 13 novembre 1936.

62. ANQ (SMC), B4-D4, Émile Nadeau au frère André Dumas, curé de Beaudry, Québec, le $1^{\mathrm{er}}$ février 1937. Ce curé demandait depuis longtemps les services d'un médecin pour ses paroissiens.

63. Jusque-là, la correspondance du SMC avait porté tantôt l'en-tête du SAP, tantôt celle du SPH, comme le révèle l'étude de Claire Martin, op. cit., 13. En ce qui concerne le budget, le docteur Martel recevait du directeur du SPH la directive suivante en date du 3 avril 1936: «Quant à vos dépenses se rapportant aux soins médicaux aux colons, vous les chargerez à un compte spécial séparé [de celui pour l'unité sanitaire], et nous avons demandé au département du Trésor une avance de $150.00 \$$ sur les Fonds de l'Assistance publique [...]» ANQ (US), B1-D1, Alphonse Lessard à Émile Martel, Québec, 3 avril 1936. 
de la Province, comme en témoigne alors le papier en-tête du SMC, les responsables de ce service pour indigents devaient, au moins une fois par année, en démontrer l'urgence et la nécessité, afin d'obtenir l'argent nécessaire à son maintien et à son expansion. Cette obligation a donné lieu à de nombreuses lettres et à des mémoires de la part du sous-ministre Grégoire surtout, qui ont permis de faire voter des budgets spéciaux provenant du fonds consolidé de la province. Ces budgets spéciaux deviennent plus fréquents dans le contexte de la Deuxième Guerre mondiale. En avril 1939, une somme de $25000 \$$ était prévue aux fins des «Dispensaires de gardes-malades ${ }^{64} \gg$. À la suite de requêtes du docteur Grégoire, un arrêté en conseil fut adopté le 16 juillet et un second le 9 octobre 1941. Dans le premier, on peut lire que le budget voté était puisé depuis plusieurs années à même le fonds de l'Assistance publique ${ }^{65}$. Cet arrêté en conseil aura permis de maintenir 24 dispensaires en Abitibi, 13 au Témiscamingue, 6 au Saguenay et 10 autres répartis ailleurs au Québec.

Encore en 1943, le docteur Grégoire invoquait l'urgence et la nécessité, en demandant à son supérieur que le département du Trésor ne réduise pas la subvention de $75000 \$$, afin de pouvoir répondre, entre autres, à la demande de trois curés dont les paroisses étaient sans dispensaire: «Ce sont des cas de réelle nécessité si nous ne voulons pas risquer de recevoir la démission de ces infirmières surtout à l'approche de la saison rigoureuse ${ }^{66}$.» Cette obligation de démontrer constamment l'importance des besoins a favorisé une gestion consistant à réagir aux pressions des milieux plutôt qu'à planifier les besoins, ce dont se plaignait le docteur Martel ${ }^{67}$. Malgré ces contraintes et tergiversations, le SMC prit de l'expansion. La période de la guerre fut à cet égard la plus favorable, alors que 72 nouveaux postes, représentant $41,37 \%$ de l'ensemble des postes créés entre 1932 et 1975, étaient autorisés ${ }^{68}$. C'est également pendant cette brève période que le Service trouvait sa niche au sein du ministère de la Santé.

64. Loi octroyant à Sa Majesté les deniers requis pour les dépenses du gouvernement pour les années financières expirant le 30 juin 1939 et le 30 juin 1940, et pour d'autres fins du service public, sanctionnée le 28 avril 1939, 3 George VI, Chapitre 1, Cédule A.

65. Arrêté en conseil, Chambre du conseil exécutif 1809, Québec, le 16 juillet 1941.

66. ANQ (SMC), B1-D1, Mémoire pour l'honorable Monsieur Groulx par le docteur Jean Grégoire, sous-ministre de la Santé, le 21 octobre 1943.

67. ANQ (SMC), B1-D1, Émile Martel, médecin hygiéniste régional, à Jean Grégoire, Amos, 22 juillet 1941.

68. Le réseau des dispensaires-résidences fera l'objet d'un article distinct. La reconnaissance du SMC dans les structures du ministère dans la période de guerre tient aussi à l'orientation, alors plus affirmée des pouvoirs publics, en faveur de l'adoption de politiques économiques et sociales sur une base régulière, sinon permanente. 


\section{Structuration de «la solution la plus raisonnable»}

En février 1936, la nouvelle structure commençait à prendre forme puisque, le 17 de ce mois, le docteur Martel adressait simultanément au docteur Lessard et «à toutes les Infirmières et Médecins actuellement dans le Service chez les colons» une lettre où il informait d'abord les intéressés du statut du SMC «pas encore organisé définitivement sur une base permanente». Il s'y identifiait comme étant chargé «de la mise en marche de ce nouveau service» dont il décrivait sommairement le fonctionnement par le biais de l'utilisation que les destinataires devaient faire des formulaires S.M.C. 1, 2, 3, 4 et $5^{69}$.

Dès ce moment, les infirmières recevaient un exemplaire des formulaires d'usage du service dont les trois premiers «entreront en force immédiatement». Un examen du formulaire SMC1 révèle qu'il constituait un outil d'application de l'article 17 de la Loi de l'assistance publique, c'està-dire qu'il visait à attester qu'une personne, jugée indigente, devait être hospitalisée aux frais du SAP. La reproduction de ce formulaire ci-dessous permet de mieux cerner le statut et le fonctionnement très particuliers du SMC.

$\begin{aligned} & \text { Secrétariat de la Province } \\ & \text { Service de l'assistance publique } \\ & \text { Hôtel du gouvernement } \\ & \text { Québec }\end{aligned}$
Canton, Colonie no.
Date:
Aux autorités de l'hôpital de
Veuillez accepter pour traitement aux frais du Service de l'Assistance publique,
M souffrant de (diagnostic probable).
signé: (médecin ou garde-malade)
Une copie de cette demande d'hospitalisation doit être transmise en même temps au
service de l'Assistance publique à Québec ainsi qu'au Dr Martel à Amos.

69. ANQ (SMC), B2-D2, Émile Martel, Lettre circulaire, non datée, annexée à celle du docteur Émile Martel, officier médical, au docteur Alphonse Lessard, directeur du SPH, Amos, 17 février 1936; ANQ (AT), Émile Martel à Jeanne Chabot, Infirmière, Sainte-Gertrude, Abitibi, 17 février 1936. 
Les coûts d'hospitalisation des colons, à l'instar de ceux des indigents, étaient ainsi assumés par le SAP; précisons qu'aux fins d'application de la Loi de l'assistance publique, le secrétaire de la Province agissait comme ministre. $\mathrm{Ce}$ formulaire permettait aux infirmières de colonies non seulement de faire hospitaliser un colon, aux frais de l'État, mais encore de poser un diagnostic préalable! Le formulaire SMC2 concernait la sortie de l'hôpital. Le formulaire SMC3, le plus important du Service, consistait en une liste d'activités accomplies par les infirmières et regroupées sous douze rubriques: 1) hygiène maternelle, 2) hygiène de la première enfance, 3 ) hygiène scolaire, 4) maladies contagieuses, 5) tuberculose, 6) vaccinations/immunisations, 7) médecine générale, 8) petites chirurgies, 9) soins dentaires, 10) obstétrique, 11) visites du médecin, et 12) hospitalisation. Pendant des décennies, il a servi aux infirmières à produire les rapports hebdomadaires qu'elles devaient adresser conjointement aux docteurs Martel, à Amos, et Lessard, à Québec. Il nous apparaît clair que l'ensemble des interventions posées par les infirmières et rapportées sous ces rubriques dépassait les limites de la «nécessité absolue» pour les populations desservies aussi bien que les attributions courantes des praticiennes. Les autorités publiques sanctionnaient ainsi la mise en place d'un réseau de services de santé étendu, pratiquement gratuit et disponible sur place. Quant aux formulaires SMC4 et SMC5, le docteur Martel se contentait de mentionner que «Ce sont les rapports des médecins». Nous n'en avons trouvé qu'un seul exemplaire dans les archives.

Le 12 novembre 1936, la loi créant le ministère de la Santé et du Bienêtre social regroupait dans une même structure administrative le SPH [santé] et le SAP [bien-être] en permettant à son titulaire: «De favoriser, par tous les moyens et mesures qu'il juge adéquats, l'avancement et le développement de la santé publique dans la province ${ }^{70}$.» Jusqu'à la création de ce ministère, les infirmières de colonies ont été embauchées par le docteur Lessard, au double titre de directeur du SAP et du SPH, puis par le docteur Nadeau, directeur intérimaire du ministère et enfin, par le docteur Grégoire, sous-ministre de la Santé de 1937 à 1962. Dans l'organigramme du ministère en 1938, le SMC, placé sous l'autorité du sous-ministre, figurait sous la rubrique «Secours médical», laquelle était subdivisée en deux volets: «Indigents de la CôteNord», on se souviendra des premiers postes créés dans cette région, et «Colons ${ }^{71} »$.

70. Loi concernant le département de la santé et du bien-être social, Statuts refondus de la Province de QuĖbec, 1941, chapitre 182, Section I, article 3. Pour plus de commodité et parce que la documentation consultée y réfère toujours en ces termes à partir de 1936, nous parlerons plutôt de «ministère de la Santé».

71. Esdras Minville, op. cit. 


\section{Des tâtonnements aux règlements}

Avec la création du ministère de la Santé, les conditions d'embauche et de travail des infirmières de colonie se «normalisèrent», d'abord dans une lettre d'embauche type, formulée en six points tenant lieu de contrat, puis dans des règlements dont la première édition remonte à avril 1942 et la dernière à février 1956. Mais malgré certains efforts pour uniformiser les procédures et les conditions de travail des infirmières, ces dernières ont souvent subi les contrecoups de problèmes imprévus.

La lettre type informait d'abord l'infirmière de sa nomination «par l'honorable ministre de la Santé et du Bien-être Social pour faire du travail d'hygiène publique et donner certains soins médicaux aux colons indigents» et précisait la date d'entrée en fonction, de même que le nom de la colonie qui lui était attitré $\mathrm{e}^{72}$. Au premier point du «contrat» figure le salaire mensuel; l'infirmière y est aussi informée des responsabilités du ministère de la Colonisation, déjà évoquées. Ensuite, le contrat stipulait qu'elle disposerait d'instruments de chirurgie «ainsi que d'un certain nombre de médicaments usuels», avec une mise en garde «qu'il ne faut pas faire d'extravagance en ce qui concerne les médicaments». Au point 3, l'infirmière apprenait qu'elle devait faire payer les médicaments, selon les moyens financiers de chacun, et remettre l'argent perçu au docteur Martel $^{73}$. La lettre décrivait ensuite les précautions à prendre pour éviter les abus de la part de la population:

[...] vous devrez, par l'entremise du curé ou autrement, faire savoir à la population que vous desservez que vous serez à sa disposition pour les consultations chez vous l'avant-midi pour tous les patients qui ne sont pas retenus au lit et capables, par conséquent, de se rendre à votre résidence. Pour les patients que vous devrez visiter à domicile l'aprèsmidi, il faudra aussi avertir la population d'éviter les appels de nuit, lorsqu'il n'y a pas urgence absolue et bien faire comprendre par tous les moyens dont vous pourrez disposer, que nous pourrons retirer votre infirmière en n'importe quel temps, si nous constations qu'il y a des abus incontrôlables ${ }^{74}$.

72. Voir entre autres les lettres d'embauche de Marguerite Turgeon (18 septembre 1936), Marcelle Gingras (30 décembre 1936), Jeannette Beaumier $(26$ mai 1937) et Irène Adam (7 décembre 1942) disponibles aux ANQ-AT.

73. ANQ-AT, Émile Nadeau, directeur intérimaire, ministère de la Santé, à Marcelle Gingras, infirmière au Canton Castagnier, Québec, 30 décembre 1936.

74. ANQ-AT, Jean Grégoire à Irène Adam, infirmière à Saint-Nazaire de Berry, Québec, 7 décembre 1942 
Au point 5 de la lettre, on demandait à l'infirmière d'adresser au docteur Martel un rapport hebdomadaire de ses activités. Le sixième point consistait en une recommandation formelle de «vous efforcer de réduire au strict nécessaire l'hospitalisation des patients». Ce dernier point de la lettre d'embauche est particulièrement intéressant car, dans le but d'être plus en mesure de juger de l'urgence et de la nécessité d'une hospitalisation, les autorités en sont venues à demander explicitement aux infirmières de poser un diagnostic médical. Ainsi, en avril 1936, le directeur du SPH demanda au docteur Martel d'avertir toutes les infirmières de donner plus de détails lorsqu'elles envoyaient un de leurs patients à l'hôpital ${ }^{75}$. Cette consigne est devenue un article des Règlements du Service Médical aux Colons encore en vigueur en 1956: «Les infirmières du service médical aux colons ne doivent pas remplir de formule de demande d'hospitalisation, à moins d'avoir vu le patient et d'avoir ébauché un diagnostic préalable $^{76} . »$

Les infirmières devaient découvrir, souvent à leurs dépens, que ces règlements étaient plus faciles à rédiger qu'à appliquer. La correspondance échangée entre les infirmières et les autorités concernées fait état des multiples problèmes: insalubrité du logement, problèmes d'approvisionnement de toutes sortes notamment en bois et en eau potable, difficultés de se faire rembourser des dépenses encourues pour le transport des malades ou les médicaments administrés, etc. Le ministère de la Santé, considérant que le ministère de la Colonisation négligeait ses obligations, prenait à sa charge à partir de 1938-1939 de nouveaux frais, comme ceux liés à la construction et à l'entretien des dispensaires ou le logement des infirmières ${ }^{77}$. Le docteur Martel faisait alors à son supérieur un aveu de la piètre qualité des dispensaires construits antérieurement ${ }^{78}$. Mais les infirmières n'étaient pas toujours mieux traitées par le ministère de la Santé que par celui de la Colonisation. À titre d'exemple, en 1938, on refusait de rembourser les frais de transport que Marie-Louise Gagnon réclamait pour un cas extraordinaire. Elle avait dû se déplacer trois fois pour un accouchement dans une famille très pauvre dont la mère souffrait

75. ANQ (SMC), B4-D9, Alphonse Lessard à Émile Martel, Québec, 9 avril 1936.

76. Armand Laberge, assistant sous-ministre suppléant, directeur par intérim, SMC, Règlements et directives à l'usage des employés du Service Médical aux Colons, Ministère de la Santé de la province de Québec, $1^{\text {er }}$ février 1956, article 61, 9.

77. ANQ (SMC), B1-D5, Jean Grégoire à Émile Martel, Québec, 2 juin 1939. Un document de quatre pages non daté intitulé Devis devant servir à l'estimé des dispensaires projetés dans les colonies est disponible aux ANQ-AT; il décrit les exigences que les contracteurs devaient respecter pour la construction des fondations, de la cave et de la maison proprement dite, incluant un croquis de la «salle de bain et système à eau chaude».

78. ANQ (SMC), B 1-D5, Émile Martel à Jean Grégoire, Amos, 10 mai 1939. 
de troubles psychologiques et le père était épileptique; des sacs de patates leur servaient de couvertures. L'infirmière avait dépensé plus de $8 \$^{79}$. Malgré l'appui du docteur Martel, le sous-ministre répondit qu'il lui était impossible de rembourser cette facture ${ }^{80}$. Avec le temps, les infirmières apprirent à faire autoriser au préalable et au cas par cas le transport des malades; par exemple, celles placées dans un village situé entre Forestville et Baie-Comeau ont souvent demandé par télégramme l'autorisation de transporter des malades à l'hôpital par avion ou par bateau ${ }^{81}$.

La conduite des autorités en regard des médicaments était tout aussi ambiguë, si bien que des infirmières accumulèrent des comptes impayés pour les médicaments qu'elles devaient elles-mêmes acheter et se faire livrer par les grandes pharmacies des centres urbains. Cette situation devenait particulièrement frustrante en 1939 , alors que les infirmières voyaient leur salaire mensuel réduit de $20 \%$, une mauvaise nouvelle que le ministère justifiait par l'augmentation plus rapide des postes de colonies que des budgets alloués au Service, ce qui fit réagir Murielle Lemieux: «J'accuse réception de votre lettre circulaire concernant le salaire de $\$ 80$ aux infirmières de colonie. [...] je tiens à vous faire savoir que les colons sont souvent dans l'impossibilité de payer les remèdes, soins médicaux et le transport. Le produit de mon travail ne suffit pas pour faire face aux dépenses ${ }^{82} \gg \ldots$

En 1943, le docteur Martel, directeur du SMC devenu une division du ministère de la Santé et déménagé à Québec, produisait son premier rapport annuel qui ne portait toutefois que sur les dispensaires de l'AbitibiTémiscamingue. On peut y lire que «L'année 1943 fut une année d'organisation générale pour le Service Médical aux Colons» et qu'il y avait, pour cette seule région, 39 postes du SMC dont «Les titulaires étaient 38 gardes-malades et 1 médecin $^{83}{ } \gg$. Ce Service infirmier, d'abord conçu pour répondre aux besoins de la principale région de colonisation pendant la crise, étendait alors son réseau dans toutes les régions éloignées de la province.

79. ANQ (SMC), B4-D9, Marie-Louise Gagnon, infirmière à Beaucanton, à Émile Martel, Beaucanton, 11 septembre 1938.

80. ANQ (SMC), B4-D9, Jean Grégoire à Émile Martel, Québec, 3 octobre 1938.

81. ANQ (SMC), B3-D1, Télégramme de Louise Matte, infirmière à Sainte-Thérèse-duColombier, à Jean Grégoire, 12 février 1941 à 4h45 p.m.); Télégramme de Louise Matte à Jean Grégoire, 22 septembre 1941 à $20 \mathrm{~h} 05$.

82. ANQ (SMC), B4-D5, Murielle Lemieux à Jean Grégoire, Rollet, 20 mars 1939.

83. Docteur Émile Martel, Directeur, Division du Service Médical aux Colons, Premier rapport annuel, dans Deuxième rapport du ministère de la Santé pour les années 1941, 1942 et 1943, Province de Québec, Ministère de la Santé et du Bien-être social, 306 et ss. 


\section{CONCLUSION}

La nécessité est la mère de l'invention. Ce dicton se confirme dans le cas de la création du Service médical aux colons. Soumises constamment aux demandes des prêtres colonisateurs et des colons eux-mêmes, contraintes par une législation qui réservait aux médecins l'exclusivité de certaines interventions qu'ils ne pouvaient d'ailleurs poser du fait de leur absence, limitées par des budgets de grande crise économique, les autorités ont quand même su trouver un compromis acceptable par toutes les parties concernées.

Mettant à profit la marge de manœuvre que leur laissaient certaines dispositions des lois en vigueur et s'inspirant des dispensaires de soins introduits dans les villes et bientôt financés par des fonds publics, de même que des précédents établis dans l'utilisation extensive des services des infirmières en cas de nécessité, les protagonistes du SMC ont conçu une solution novatrice, jugée raisonnable et assez satisfaisante pour qu'on décide de la maintenir bien au-delà de la crise économique. La participation des femmes, les premières visées par cette initiative, reste cependant peu connue. Quelques allusions - rares cependant contrairement à la situation de l'Alberta - au lobbying de certains groupes de femmes comme les Women's Institutes et les Cercles de fermières, nous enjoignent à pousser plus loin la recherche en ce sens. Chose certaine, le succès de cette solution reposait sur les infirmières de colonies, principales intervenantes de ce réseau. Leur présence, leur disponibilité constante, leur compétence, leur générosité et leur dévouement ont permis que des services d'assistance minimale et à des coûts acceptables soient offerts à des indigents. Par leur habileté à plaider l'urgence et la nécessité d'intervenir, elles ont fait en sorte que des milliers de pauvres vivant loin des centres urbains puissent bénéficier de services de santé qui ne leur auraient pas été autrement accessibles. 